\title{
Energy Efficiency TDMA/CSMA Hybrid Protocol with Power Control for WSN
}

\author{
Xin Yang $\mathbb{D},{ }^{1,2}$ Ling Wang $\mathbb{D}^{1},{ }^{1}$ Jian Xie, $^{1}$ and Zhaolin Zhang ${ }^{1}$ \\ ${ }^{1}$ School of Electronics and Information, Northwestern Polytechnical University, Xi'an, China \\ ${ }^{2}$ Jack Baskin School of Engineering, University of California, Santa Cruz, CA, USA \\ Correspondence should be addressed to Xin Yang; x_yang119@126.com
}

Received 21 November 2017; Revised 1 March 2018; Accepted 1 April 2018; Published 7 May 2018

Academic Editor: Juan F. Valenzuela-Valdés

Copyright (C) 2018 Xin Yang et al. This is an open access article distributed under the Creative Commons Attribution License, which permits unrestricted use, distribution, and reproduction in any medium, provided the original work is properly cited.

\begin{abstract}
Wireless sensors network (WSN) is widely used in the Internet of Things at present. However, limited energy source is a critical problem in the improvement and practical applications of WSN, so it is necessary to improve the energy efficiency. As another important evaluation criterion of transmission performance, throughput should be improved too. To mitigate both of the problems at the same time, by taking the advantages of Time Division Multiple Access (TDMA) and Carrier Sense Multiple Access (CSMA) at the medium access control (MAC) layer of WSN, we propose a hybrid TDMA/CSMA MAC layer protocol. Meanwhile, we design a novel power control scheme to further reduce the energy consumption and optimize the transmission slots. The simulation results demonstrate that the proposed protocol significantly improves the throughput and energy efficiency.
\end{abstract}

\section{Introduction}

Wireless sensors network (WSN) suffers from energy efficiency problem in many applications. It is crucial to improve the energy efficiency to extend the lifetime of WSN [1]. Many efforts have been made by researchers to enhance the energy consumption performance, most of which are in the physical (PHY) layer of the networks [2]. Besides PHY layer, MAC layer also has a critical influence on the energy consumption performance of WSN $[3,4]$. However, it is difficult to reduce energy consumption while improving the transmission performance. As is widely used in the Internet of Things (IoT), vehicle ad hoc networks (VANET), and other wireless communication systems, WSN is urgently required to enhance energy efficiency, throughput, and other transmission performance $[5,6]$.

In recent years, many protocols based on TDMA and CSMA mechanism are proposed to enhance the energy efficiency of WSN. However, TDMA scheme wastes slots resources in light traffic networks, while the contending scheme of CSMA is liable to cause collisions in high traffic. For instance, a TDMA-based protocol is described in [7] to decrease energy consumption in WSN, but it also has a negative influence on the transmission performance as the throughput. Meanwhile, another MAC layer protocol named Energy-FDM is proposed based on CSMA in [8]; it reduces energy consumption by transmission power control, but it leads to a high collision rate, especially in high traffic networks. The pTunes [9] saves energy by adapting its own MAC parameters on the channel models, but the network information collection scheme results in transmission delay. Considering the defects of the protocols above, on the other side, some hybrid scheme protocols are proposed to save energy in WSN. OPWUM [10] makes efforts on receivers with leveraging wake-up hybrid scheme and reduces energy consumption effectively, but the frame structure design is complicated and leads to a waste of resources, so it has a negative influence on transmission performance. Another novel multihop MAC protocol CEE [11] aims at reducing energy consumption based on cross-layer transmission model with hybrid transmission scheme. However, the collision rate is high due to the channel allocation design and affects the throughput. As a consequence, we propose a TDMA/CSMA hybrid MAC layer protocol (TCH-MAC) to enhance the energy efficiency of WSN. The TDMA scheme in the protocol is a traffic adaptive TDMA (TA-TDMA) based on reservation to handle traffic bursting problem. The CSMA scheme that we use in TCH-MAC is an efficient CSMA (E-CSMA). We also 


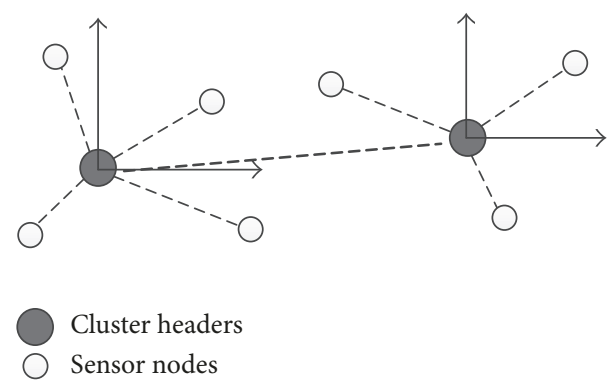

FIGURE 1: The cluster structure of WSN.

utilize adjustable transmission power control scheme in the proposed protocol. The analysis and simulation results show that our protocol not only improves the energy efficiency, but also enhances the transmission throughput.

The rest of this letter is organized as follows. We present the details of proposed protocol in the second section, with transmission slots design and power control scheme. The third section shows the analysis of TCH-MAC. Then the simulation results are presented in the fourth section on throughput and energy consumption. Finally, conclusion is made in the fifth section.

\section{TCH-MAC Protocol}

In this section, we describe the proposed TCH-MAC protocol based on TDMA/CSMA hybrid mechanism in detail. With reservation based TA-TDMA that we utilize, the wake-up scheme effectively controls the sleeping and wake-up time of nodes to reduce the energy consumption. We also describe the slots frames of the proposed protocol, based on which we then describe the power control scheme.

Consider a cluster WSN system as shown in Figure 1, which is divided into many clusters; each cluster has a cluster header $(\mathrm{CH})$ and some sensor nodes (SNs). All the SNs can transmit data packets to their own $\mathrm{CH}$. A CH can communicate with another $\mathrm{CH}$ in the WSN system. The transmission between two $\mathrm{CHs}$ is called cluster to cluster transmission (CCT) in the proposed protocol.

2.1. SN to CH Transmission. The main function of SNs in WSN is collecting surrounding information and transmitting to backstage server. Hence, we describe the details of transmission from $\mathrm{SN}$ to its $\mathrm{CH}$. Also, the energy is mainly consumed in the transmitting from SNs to their $\mathrm{CH}$ among all transmission period, which is crucial to the lifetime of WSN. The transmission protocol between a $\mathrm{CH}$ and a fixed SN consists of three periods, namely, contending period (CP), TA-TDMA period (TP), and sleeping period (SP), respectively. The details are shown in Figure 2, where the synchronization (Sync) and CCT period (CTP) are used for communicating with another $\mathrm{CH}$.

2.1.1. CP of TCH-MAC. The main function of this period is contended for the transmission order in TA-TDMA period for saving energy. As shown in Figure 3, every node sends a request to send (RTS) packet after a random backoff at the beginning of CP. Because different nodes have different backoff values, the first one successfully delivers RTS to $\mathrm{CH}$ which can communicate with $\mathrm{CH}$. The overstriking in Figure 3 is SIFS (Short Interframe Space), which lies in every switch of transmitting and receiving period of SN. In Figure 3, we assume that SN 1 is the first access one. Other nodes send RTS and find that cluster is busy, so they turn off and switch to sleeping period. The transmission in this period between $\mathrm{CH}$ and SN based on CSMA is E-CSMA. There are three differences between E-CSMA and traditional CSMA. Firstly, only one data packet can be transmitted in data transmission (DATA) period; other packets are transmitted in TP. Secondly, the acknowledgement (ACK) in CSMA is replaced by acknowledgement and information (ACI) packet, which is described as the first line of Figure 4 in detail. It includes ACK packets, the information about number of nodes, nodes' ID, location, and data length. The ACI is updated after every DATA transmission and broadcasts to all nodes in the cluster. For example, we assume the numbers of data packets that need to be transmitted by SN 1, SN 2, and SN 3 are 3, 2, and 4, respectively. Because one packet has already been transmitted in CP, the values of data length at the end of CP are $l_{1}=2, l_{2}=1$, and $l_{3}=3$, as shown in the second line of Figure 4. According to the latest ACI, CH can allocate TA-TDMA period. Thirdly, the E-CSMA complies with adjustable transmission power scheme, which will be presented later.

When the $\mathrm{CH}$ broadcasts $\mathrm{ACI}$, all of the nodes in this cluster wake up and receive it. Then they will send RTS again after a random backoff. Like Figure 3, the SN 3 gets the opportunity to communicate with $\mathrm{CH}$. The steps are as same as those of SN 1. Meanwhile, SN 1 and SN 2 turn off and go into sleeping period. Finally, the SN 2 finishes the transmission with the $\mathrm{CH}$. The ACI can also inform all nodes about their schedule details in TA-TDMA.

2.1.2. TA-TDMA of TCH-MAC. The processing of TA-TDMA period is shown in Figure 5; we also take the 3 nodes in the last subsection as an example. The $\mathrm{CH}$ first broadcasts a beacon in TA-TDMA with two purposes: one is waking up the sleeping SNs; the other is synchronized with other nodes. Then the three nodes send data packets to the $\mathrm{CH}$ according to the order ranked in CP. When there is no packet to send, SNs turn off to sleep. After all the data packets are transmitted to the $\mathrm{CH}$, the protocol process switches to CTP.

2.1.3. CCT Period of TCH-MAC. The CTP is for two CHs' communication. Take $\mathrm{CH} 1$ and $\mathrm{CH}$ 2, for example. The first $\mathrm{CH}$ that finishes TA-TDMA period starts to sleep until receiving Sync by another one. Sync is used for waking up and synchronization between two CHs. As shown in Figure 6, CH 1 sends a Sync to $\mathrm{CH} 2$, including synchronization information, location information, and waking up information. Then, $\mathrm{CH} 2$ sends data packets to $\mathrm{CH} 1$ end with finish information (FI), attached with its location information. Next, $\mathrm{CH} 1$ transmits its data packets to $\mathrm{CH} 2$ after receiving the FI from $\mathrm{CH} 2$, also ending with FI. Finally, this frame is finished. In addition, the multiple $\mathrm{CHs}$ communication processing is the 


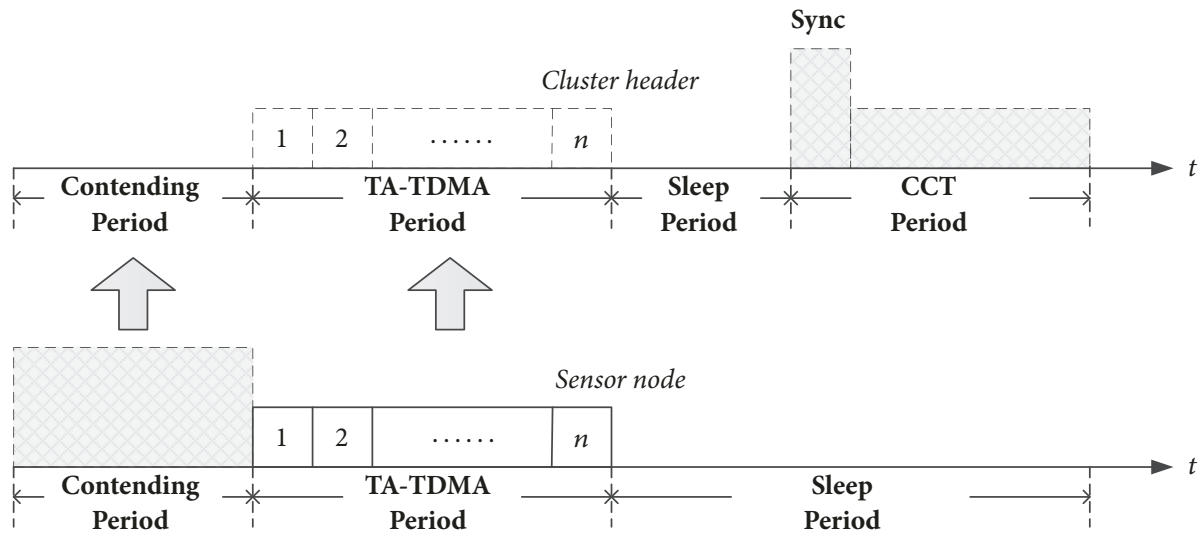

FIGURE 2: The transmission scheme between $\mathrm{SN}$ and $\mathrm{CH}$.
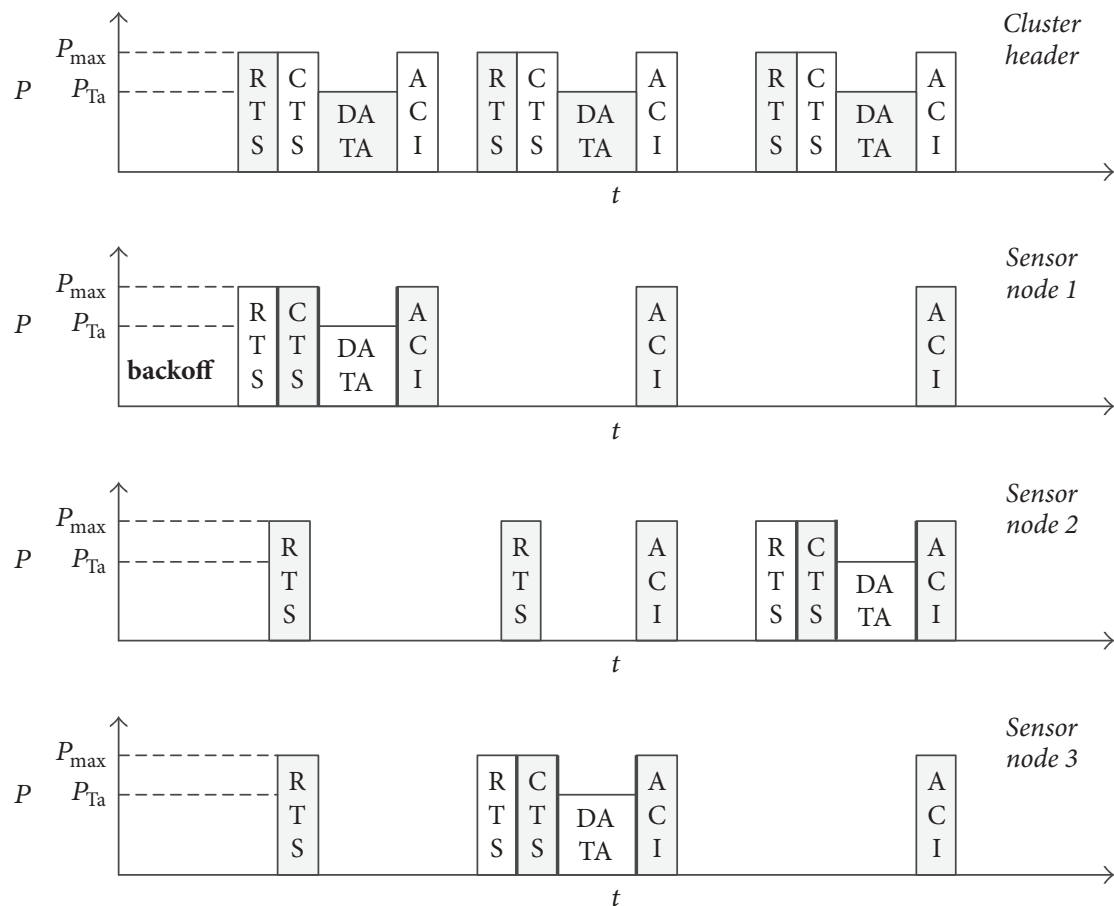

Transmit packet

Received packet

Figure 3: CP of TCH-MAC based on E-CSMA.

\begin{tabular}{|c|c|c|c|c|c|c|c|c|}
\hline ACK & Ely & $\begin{array}{l}x_{1} \\
y_{1} \\
\end{array}$ & $\begin{array}{l}x_{2} \\
y_{2} \\
\end{array}$ & & $\begin{array}{l}x_{n} \\
y_{n}\end{array}$ & $l_{1}$ & $l_{2}$ & $l_{n}$ \\
\hline ACK & 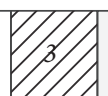 & $\begin{array}{l}x_{1} \\
y_{1}\end{array}$ & $\begin{array}{l}x_{3} \\
y_{3}\end{array}$ & $\begin{array}{l}x_{2} \\
y_{2}\end{array}$ & $l_{1}$ & $l_{3}$ & $l_{2}$ & \\
\hline
\end{tabular}

Number of nodes

Nodes ID/location

Data length

Figure 4: Details of ACI. 


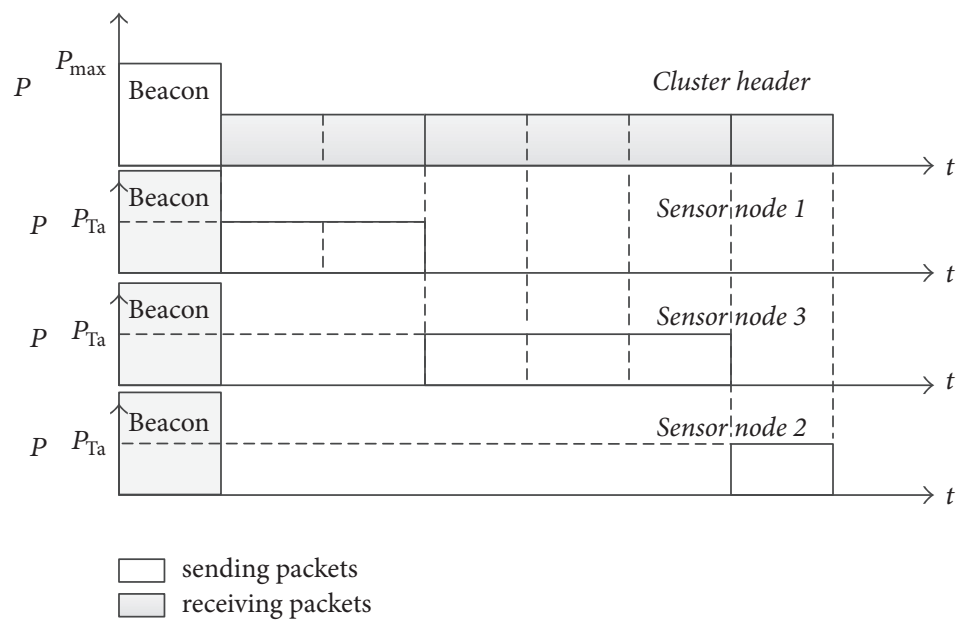

FIgURE 5: TA-TDMA scheme in TCH-MAC.

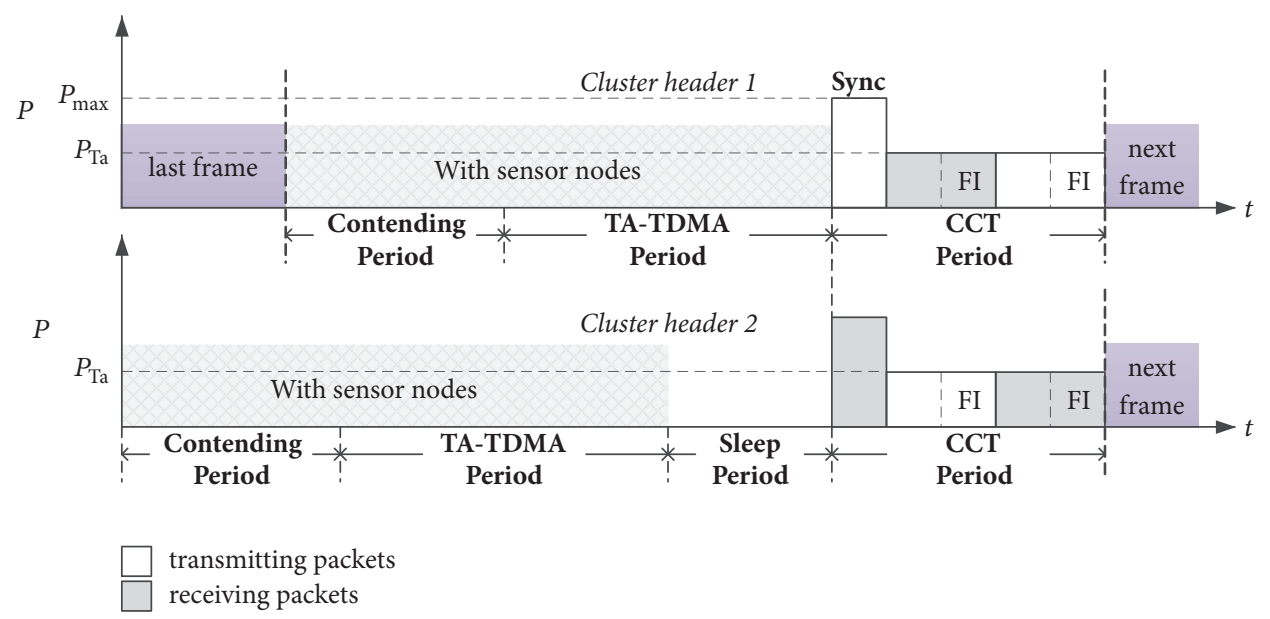

Figure 6: CCT period scheme in TCH-MAC.

repetition of two CHs; they are in the same way. For example, when $\mathrm{CH} 1$ wants to communicate with $\mathrm{CH} 3$, the first step is from $\mathrm{CH} 1$ to $\mathrm{CH} 2$ and then from $\mathrm{CH} 2$ to $\mathrm{CH} 3$.

2.2. Power Control Scheme. In this subsection, we describe the power control scheme of TCH-MAC. In different slots, different transmitting power values are designed for SNs and CHs. The $y$-axis in Figures 2, 3, 5, and 6 represents transmitting power. In the proposed protocol, we design that if the transmitted packets are needed to be received by all members in the cluster, the senders should transmit them in the maximum power $P_{\max }$ to cover the transmission range area, for instance, the RTS, CTS, and ACI of CP in Figure 3, the beacon packets of TA-TDMA in Figure 5, and the Sync packets of CCT period in Figure 6. On the other hand, when the transmission is between two fixed nodes, the sender sends packets in adjustable transmitting power $P_{\mathrm{Ta}}$ to save energy. In WSN systems about transmission power, we have

$$
P_{\mathrm{Rmin}}=P_{\mathrm{Ta}}+G_{\mathrm{A}}-L_{\mathrm{E}}-L_{\mathrm{S}}
$$

where $P_{\mathrm{Rmin}}$ is the minimum necessary receiving power, $G_{\mathrm{A}}$ is the antenna gain, including transmitting antenna and receiving antenna, $L_{\mathrm{E}}$ is equipment loss, and $L_{\mathrm{S}}$ is transmission space loss. In addition, the transmission space loss is defined as

$$
L_{\mathrm{S}}(\mathrm{dB})=32.45+20 \lg F+20 \lg D \text {, }
$$

where $F(\mathrm{MHz})$ is the frequency of system; $D(\mathrm{~km})$ is the distance between sender and receiver. Because the location information is contained in ACI ( $\mathrm{SN}$ to $\mathrm{CH}$ transmission) and Sync ( $\mathrm{CH}$ to $\mathrm{CH}$ transmission), $D$ can be calculated by senders. As a result, the adjustable transmitting power in LOS (Line of Sight) condition is obtained as

$$
\begin{aligned}
P_{\mathrm{Ta}} & =P_{\mathrm{R} \min }-G_{\mathrm{A}}+L_{\mathrm{E}}+L_{\mathrm{S}} \\
& =P_{\mathrm{R} \min }-G_{\mathrm{A}}+L_{\mathrm{E}}+32.45+20 \lg F+20 \lg D .
\end{aligned}
$$


For non-LOS condition, the transmission power can be obtained by RSSI (Received Signal Strength Indicator). The average value of RSSI ( RSSI $\left._{\text {Ave }}\right)$ can be obtained as

$$
\begin{aligned}
\operatorname{RSSI}_{\text {Ins }} & =\int_{0}^{104} I^{2}+Q^{2}, \\
\mathrm{RSSI}_{\text {Ave }} & =\frac{\sum_{j=1}^{8192} \mathrm{RSSI}_{j}}{8192},
\end{aligned}
$$

where $\mathrm{RSSI}_{\text {Ins }}$ is the instant value of RSSI, I represents the inphase signal, and $Q$ stands for the quadrature signal. Depending on $\mathrm{RSSI}_{\text {Ins }}$, the transmission power in non-LOS condition can be calculated. In this paper, we mainly study LOS and MAC layer protocol. We are extending the protocol for under the non-LOS conditions with RSSI in further works.

\section{Performance Analysis}

In this section, we analyze the performance of the proposed protocol on the throughput and energy consumption.

3.1. Throughput. We assume that the probability that every node has packets to transmit in each slot is $p_{s}$ in our analysis. Taking two CHs' communication as an example, the multiple CHs can be extended in the same way as described in the second section. There are $n$ nodes in a cluster of WSN system, so the successful transmission probability in contending period is $p_{\mathrm{SC}}=n p_{s}\left(1-p_{s}\right)^{n-1}$. In CCT period, the probability that a $\mathrm{CH}$ has data packets to send is $p_{c}$. Hence, the probability that there is at least a transmission in a CCT period is

$$
p_{\mathrm{CC}}=1-\left(1-p_{c}\right)^{2}=2 p_{c}-p_{c}^{2}
$$

The time spent on CP, TP, and SP in SN to $\mathrm{CH}$ transmission is represented as $T_{\mathrm{CP}}, T_{\mathrm{TP}}$, and $T_{\mathrm{SSP}}$, respectively. Therefore, the time spent on $\mathrm{SN}$ to $\mathrm{CH}$ transmission is $T_{\mathrm{SC}}=$ $T_{\mathrm{CP}}+T_{\mathrm{TP}}+T_{\mathrm{SSP}}$.

In addition, $T_{\mathrm{CC}}=T_{\text {Sync }}+T_{\text {DATA }}+T_{\mathrm{CSP}}$ is the time spent on $\mathrm{CH}$ to $\mathrm{CH}$ transmission, where $T_{\text {Sync }}, T_{\text {DATA }}$, and $T_{\mathrm{CSP}}$ are the time costs on Sync, data transmission, and SP in $\mathrm{CH}$ to $\mathrm{CH}$ transmission.

As a consequence, the throughput of networks is defined as

$$
\begin{aligned}
H & =\frac{[\text { Successful tramsmission length }]}{[\text { Time for successful transmission }]} \\
& =\frac{p_{\mathrm{SC}} L_{\mathrm{SC}}+p_{\mathrm{CC}} L_{\mathrm{CC}}}{T_{\mathrm{SC}}+T_{\mathrm{CC}}} \\
& =\frac{n p_{s}\left(1-p_{s}\right)^{n-1} L_{\mathrm{SC}}+\left(2 p_{c}-p_{c}^{2}\right) L_{\mathrm{CC}}}{\left(T_{\mathrm{CP}}+T_{\mathrm{TP}}+T_{\mathrm{SSP}}\right)+\left(T_{\mathrm{Sync}}+T_{\mathrm{CCT}}+T_{\mathrm{CSP}}\right)},
\end{aligned}
$$

where $L_{\mathrm{SC}}$ and $L_{\mathrm{CC}}$ represent the transmitted packet length in $\mathrm{SN}$ to $\mathrm{CH}$ transmission and $\mathrm{CH}$ to $\mathrm{CH}$ transmission, respectively.
3.2. Energy Consumption. The total energy consumption $E_{\text {total }}$ includes the energy cost on $\mathrm{SN}$ to $\mathrm{CH}$ transmission and $\mathrm{CH}$ to $\mathrm{CH}$ transmission, namely, $E_{\mathrm{SC}}$ and $E_{\mathrm{CC}}$. The energy consumption of $\mathrm{CH}$ is not constrained as they can be charged easily, so we do not focus on the energy of $\mathrm{CH}$. Therefore, we obtain

$$
E_{\text {total }}=E_{\mathrm{SC}}+E_{\mathrm{CC}}=\sum_{i=1}^{n_{s}}\left(E_{i \mathrm{CP}}+E_{i \mathrm{TP}}\right)+E_{\mathrm{CC}}
$$

where $E_{i \mathrm{CP}}$ and $E_{i \mathrm{TP}}$ represent the energy that is consumed in $\mathrm{CP}$ and TP of SN to $\mathrm{CH}$ transmission of the $i$ th nodes in the cluster.

Specifically, we have

$$
\begin{aligned}
E_{i \mathrm{CP}}= & \left(T_{i \mathrm{RTS}}+T_{i \mathrm{CTS}}+T_{i \mathrm{ACI}}+T_{i \mathrm{Lis}}\right) P_{\max } \\
& +T_{i \mathrm{DATA}} P_{i \mathrm{Ta}}
\end{aligned}
$$

where $T_{i \mathrm{RTS}}, T_{i \mathrm{CTS}}, T_{i \mathrm{ACI}}, T_{i \mathrm{Lis}}$, and $T_{i \mathrm{DATA}}$ represent the time spent on RTS, CTS, ACI, listening, and DATA of $i$ th node in the cluster. $P_{i \mathrm{Ta}}$ is the adjustable transmitting power of the $i$ th node. In addition, we can obtain

$$
E_{i \mathrm{TP}}=T_{i \mathrm{~B}} P_{\max }+T_{i \mathrm{TD}} P_{i \mathrm{Ta}},
$$

where $T_{i \mathrm{~B}}$ is the beacon time of $i$ th node and $T_{i \mathrm{TD}}$ is the data transmission time of $i$ th node in TP. For $E_{\mathrm{CC}}$, we define

$$
\begin{aligned}
E_{\mathrm{CC}}= & T_{\text {Sync }} P_{h \max }+\left(T_{1 \mathrm{D}}+T_{1 \mathrm{FI}}\right) P_{1 \mathrm{Ta}} \\
& +\left(T_{2 \mathrm{D}}+T_{2 \mathrm{FI}}\right) P_{2 \mathrm{Ta}},
\end{aligned}
$$

where $P_{\mathrm{hmax}}$ is the maximum power of header, which may be different from $P_{\max }$. $T_{\text {Sync }}$ is the Sync time. We assume the two cluster headers in transmission are header 1 and header 2. $T_{1 \mathrm{D}}$ and $T_{2 \mathrm{D}}$ are the time costs on data transmission of headers 1 and 2 in CCP, respectively. In a similar way, $T_{1 \mathrm{FI}}$ and $T_{2 \mathrm{FI}}$ are the time costs on FI of headers 1 and 2 in CCP, respectively.

\section{Simulations Results}

In the simulations, we assume there are 20 to 200 nodes randomly distributed in an area of $40 \mathrm{~m} \times 40 \mathrm{~m}$. All of the nodes are divided into clusters according their distance with each other; the number of nodes in each cluster is from 5 to 10 randomly. The transmission bandwidth is $1 \mathrm{Mbps}$ and the frequency is $2.4 \mathrm{GHz}$. We set 240 bits for RTS and CTS, 320 bits for ACI, 128 for beacon and FI. The maximum transmit power of $\mathrm{CH}$ and $\mathrm{SN}$ is $26 \mathrm{dBm}$ and $24 \mathrm{dBm}$, respectively. The receiver sensitivity of all nodes is $-125 \mathrm{dBm}$. The antenna gain is offset by equipment loss in the simulations. We obtain the simulation results by NS-3.26. In section, we take the protocols Energy-FDM [8], CEE [11], and Z-MAC [5] for comparison, because they are all aiming at energy efficiency and throughput improvement for wireless networks.

The simulation results of throughput are shown in Figure 7. We note that the throughput increases with the increasing number of nodes. When the number of nodes is more than 160, the throughput increases slowly because the 


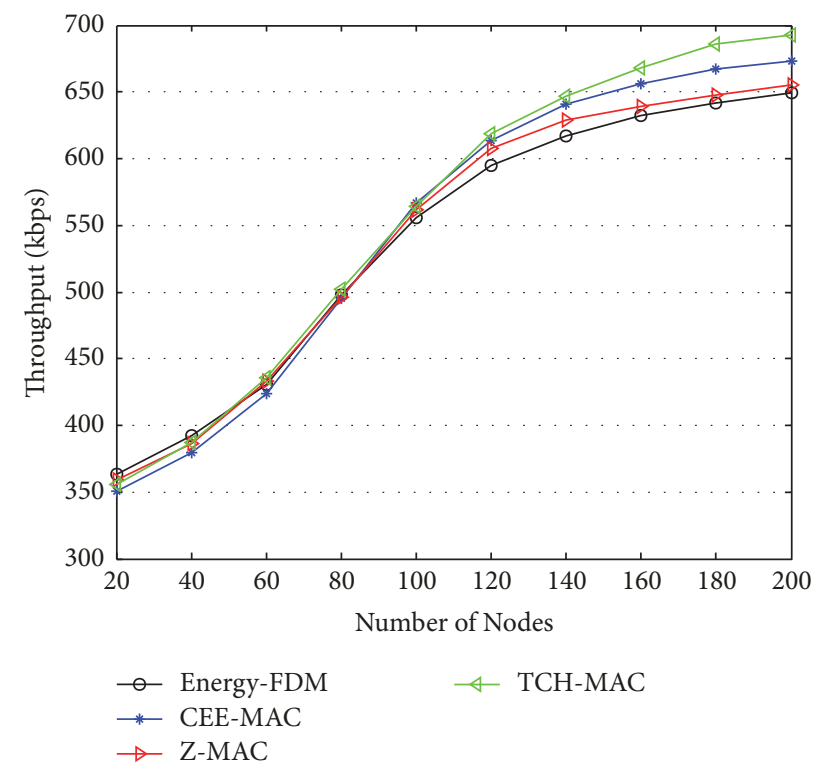

FIGURE 7: Simulation results of throughput versus number of nodes.

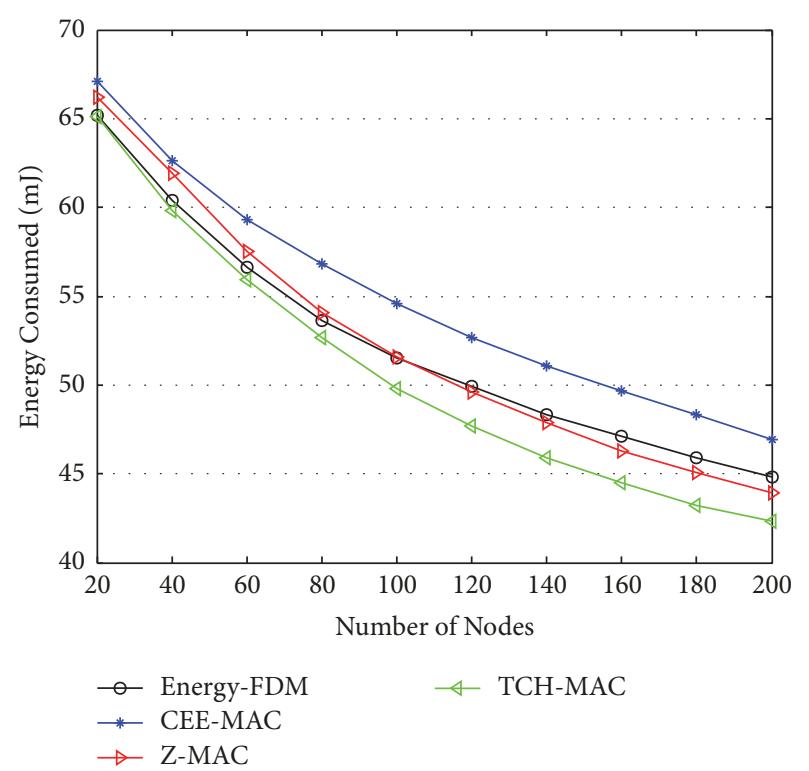

FIGURE 8: Simulation results of energy consumption versus number of nodes.

network is near to saturation. The three protocols provide a similar throughput performance when the number of nodes is smaller than 100 . TCH-MAC shows its advantage in large amount of nodes with a 3\%,6\%, and 9\% higher throughput than Z-MAC, CEE, and Energy-FDM; this is because the TDMA/CSMA hybrid scheme we utilize can efficiently allocate slots for transmission and avoid collision. TCHMAC takes full use of transmission slots by dividing the transmission into $\mathrm{CP}, \mathrm{TP}, \mathrm{SP}$, and $\mathrm{CCP}$ to improve the throughput.

Figure 8 shows the per node's energy consumption of the four protocols when $1 \mathrm{Mbit}$ data are transmitted in WSN.

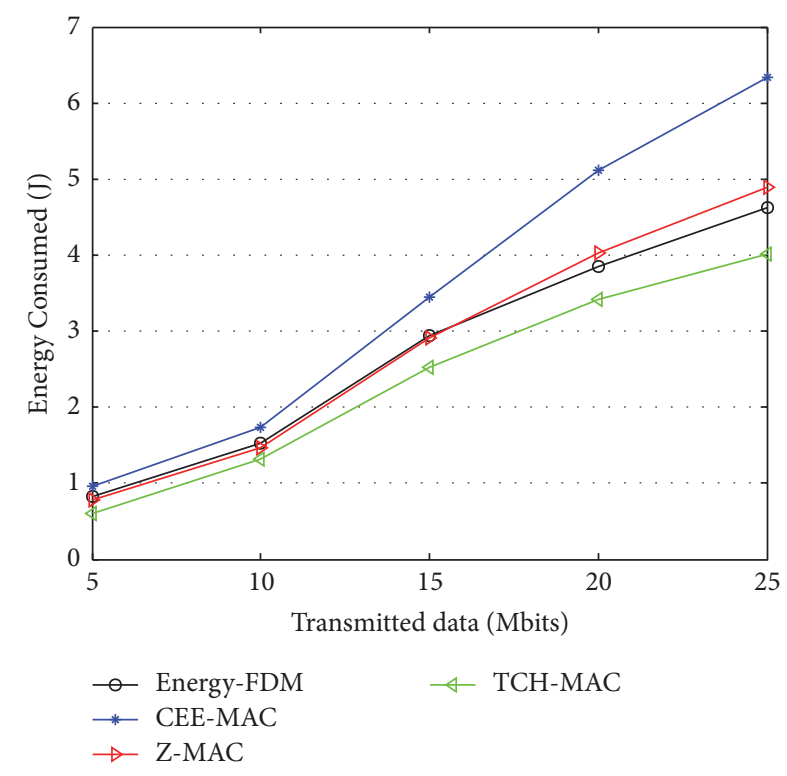

FIGURE 9: Simulation results of energy consumption versus transmitted data.

Because more nodes lead to shorter transmission links, the energy consumption reduces with the increment of nodes. TCH-MAC outperforms CEE, Energy-FDM, and Z-MAC with almost $12 \%, 9 \%$, and 5\% lower energy consumption, respectively. The main reason is adjustable transmission power control and sleeping period design can save much energy comparing to other protocols. In addition, the TDMA/CSMA hybrid scheme can make full use of time slots to save energy and prevent unnecessary waste.

Figure 9 shows the energy consumption of the four protocols when the numbers of transmitted data are different. It is clear to find that our protocol has the best performance among the four protocols. It uses almost $12 \%, 18 \%$, and $30 \%$ less energy than Energy-FDM, Z-MAC, and CEE-MAC, respectively. The advantages are more obvious when more data are transmitted. Because more transmitted data need more nodes to take part in the transmission, the distance values of $\mathrm{SN}$ s to $\mathrm{CH}$ s are diverse. Also, the TA-TDMA scheme in the proposed protocol helps save energy because it is traffic adaptive. It can take full use of every slot to transmit and thus to reduce transmission time and energy. As a result, the proposed protocol can adaptively control the transmitting power and improve the energy efficiency.

\section{Conclusion}

In this paper, we proposed a hybrid TDMA/CSMA protocol named TCH-MAC for WSN to reduce the energy consumption and enhance the transmission throughput. The proposed protocol takes advantages of TDMA and CSMA scheme in the MAC layer. The TDMA scheme in this paper is traffic adaptive and the CSMA is energy efficient. We described the details of each period in the proposed protocol, namely, CP, TP, SP, and CCT. In addition, we separately presented the transmission details from $\mathrm{CH}$ to $\mathrm{CH}$ and $\mathrm{SN}$ to $\mathrm{CH}$. 
Meanwhile, we design a novel power control scheme to further reduce energy consumption and optimize transmission slots. The analysis and simulation results of TCH-MAC are also presented to show the improvement in throughput and energy consumption of the proposed protocol.

\section{Conflicts of Interest}

The authors declare that there are no conflicts of interest regarding the publication of this article.

\section{Acknowledgments}

This study was supported in part by the National Natural Science Foundation of China (nos. 61771404, 61601372, and 61601373).

\section{References}

[1] F. Z. Djiroun and D. Djenouri, "MAC protocols with wake-up radio for wireless sensor networks: a review," IEEE Communications Surveys \& Tutorials, vol. 19, no. 1, pp. 587-618, 2017.

[2] W. Ye, J. Heidemann, and D. Estrin, "An energy-efficient MAC protocol for wireless sensor networks," in Proceedings of the 21 Annual Joint Conference of the IEEE Computer and Communications Societies, vol. 3, pp. 1567-1576, IEEE, New York, NY, USA, June 2002.

[3] S. Zhuo, Y.-Q. Song, Z. Wang, and Z. Wang, "Queue-MAC: A queue-length aware hybrid CSMA/TDMA MAC protocol for providing dynamic adaptation to traffic and duty-cycle variation in wireless sensor networks," in Proceedings of the 2012 9th IEEE International Workshop on Factory Communication Systems, WFCS 2012, pp. 105-114, IEEE, Lemgo, Germany, May 2012.

[4] A. El-Hoiydi and J. Decotignie, "WiseMAC: an ultra low power MAC protocol for the downlink of infrastructure wireless sensor networks," in Proceedings of the Proceedings. ISCC 2004. Ninth International Symposium on Computers And Communications (IEEE Cat. No.04TH8769), pp. 244-251, Alexandria, Egypt.

[5] I. Rhee, A. Warrier, and M. Aia, "Z-MAC: a hybrid MAC for wireless sensor networks," IEEE/ACM Transactions on Networking, vol. 16, no. 3, pp. 511-524, 2008.

[6] M. M. Alam, O. Berder, D. Menard, and O. Sentieys, "TADMAC: traffic-aware dynamic MAC protocol for wireless body area sensor networks," IEEE Journal on Emerging and Selected Topics in Circuits and Systems, vol. 2, no. 1, pp. 109-119, 2012.

[7] A. Amokrane, R. Langar, R. Boutaba, and G. Pujolle, "A green framework for energy efficient management in TDMAbased wireless mesh networks," in Proceedings of the 8th IEEE International Conference Network and service management, vol. 62, pp. 322-328, IEEE, Las Vegas, NV, USA, 2012.

[8] M. O. Al-Kadri, A. Aijaz, and A. Nallanathan, "An EnergyEfficient Full-Duplex MAC Protocol for Distributed Wireless Networks," IEEE Wireless Communications Letters, vol. 5, no. 1, pp. 44-47, 2016.

[9] M. Zimmerling, F. Ferrari, L. Mottola, T. Voigt, and L. Thiele, "pTunes: runtime parameter adaptation for low-power MAC protocols," in Proceedings of the 11th ACM/IEEE Conference on Information Processing in Sensing Networks (IPSN '12), pp. 173184, Beijing, China, April 2012.
[10] F. A. Aoudia, M. Gautier, and O. Berder, "OPWUM: Opportunistic MAC protocol leveraging wake-up receivers in WSNs," Journal of Sensors, vol. 2016, Article ID 6263719, 2016.

[11] X. Yang, L. Wang, and J. Xie, "Energy Efficient Cross-Layer Transmission Model for Mobile Wireless Sensor Networks," Mobile Information Systems, vol. 2017, pp. 1-8, 2017. 


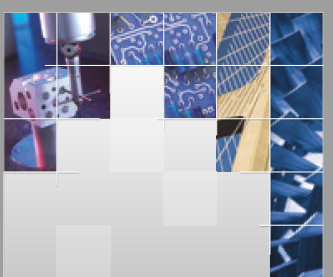

\section{Enfincering}
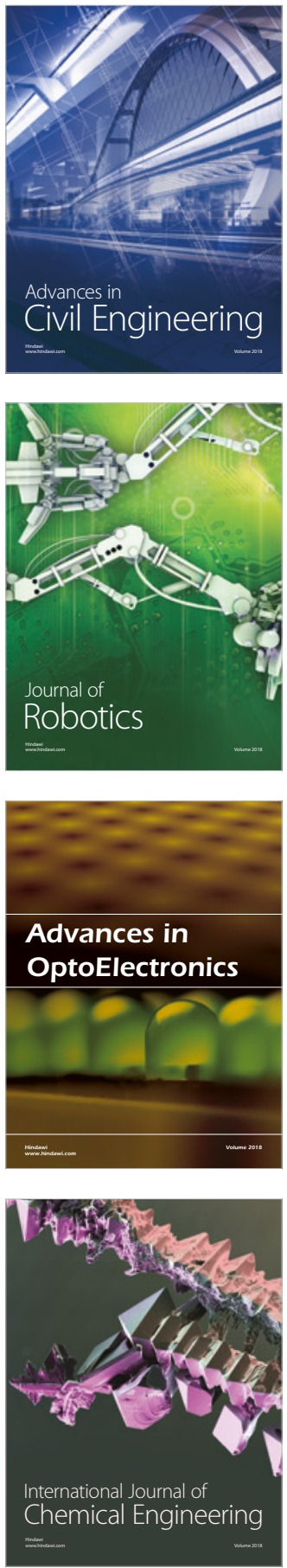

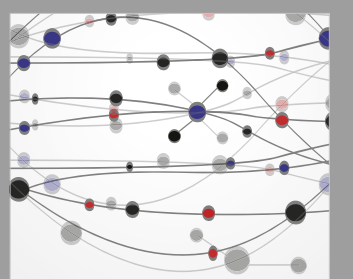

\section{Rotating \\ Machinery}

The Scientific World Journal

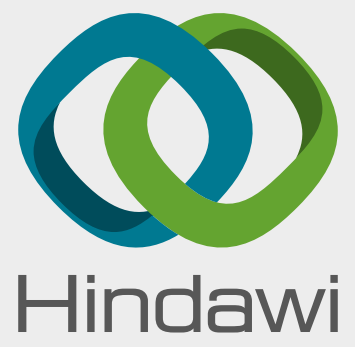

Submit your manuscripts at

www.hindawi.com
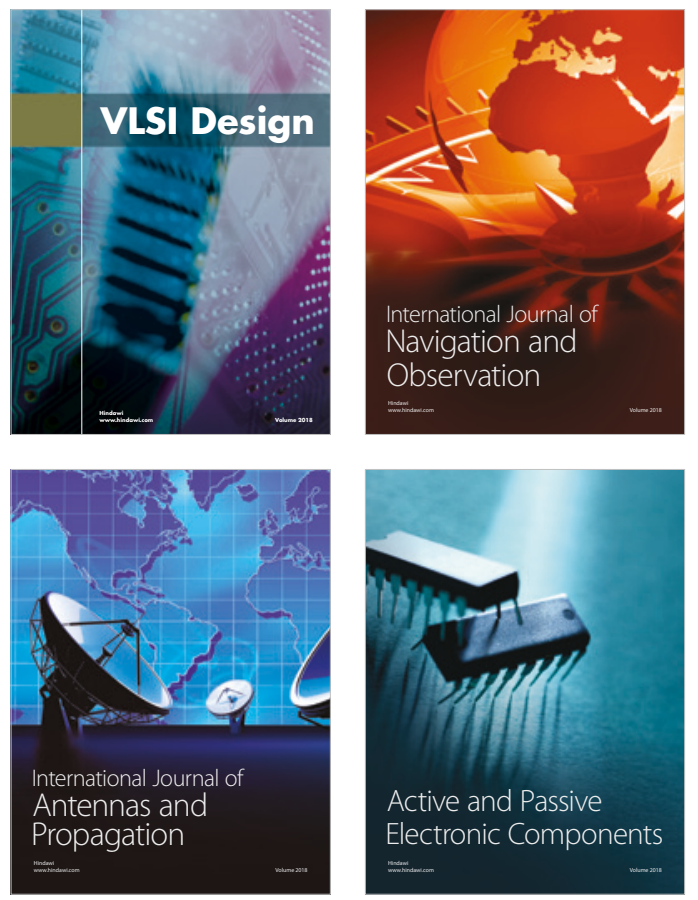
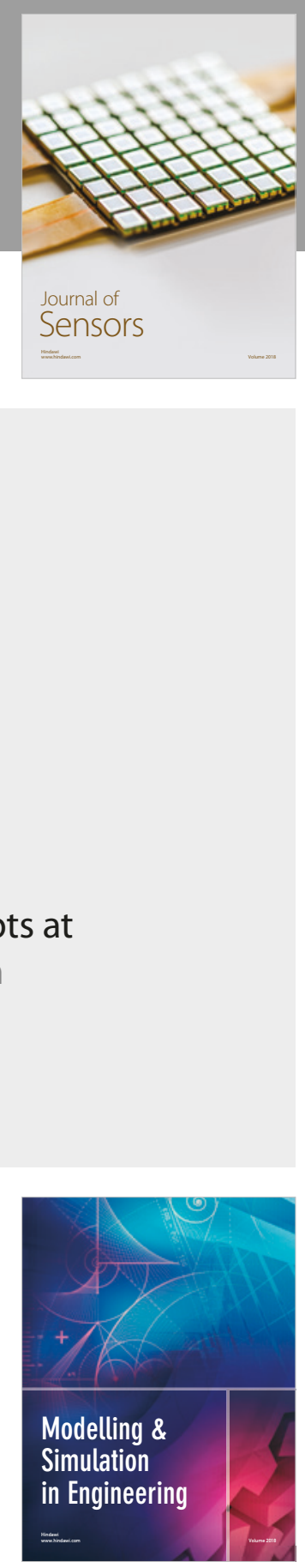

\section{Advances \\ Multimedia}
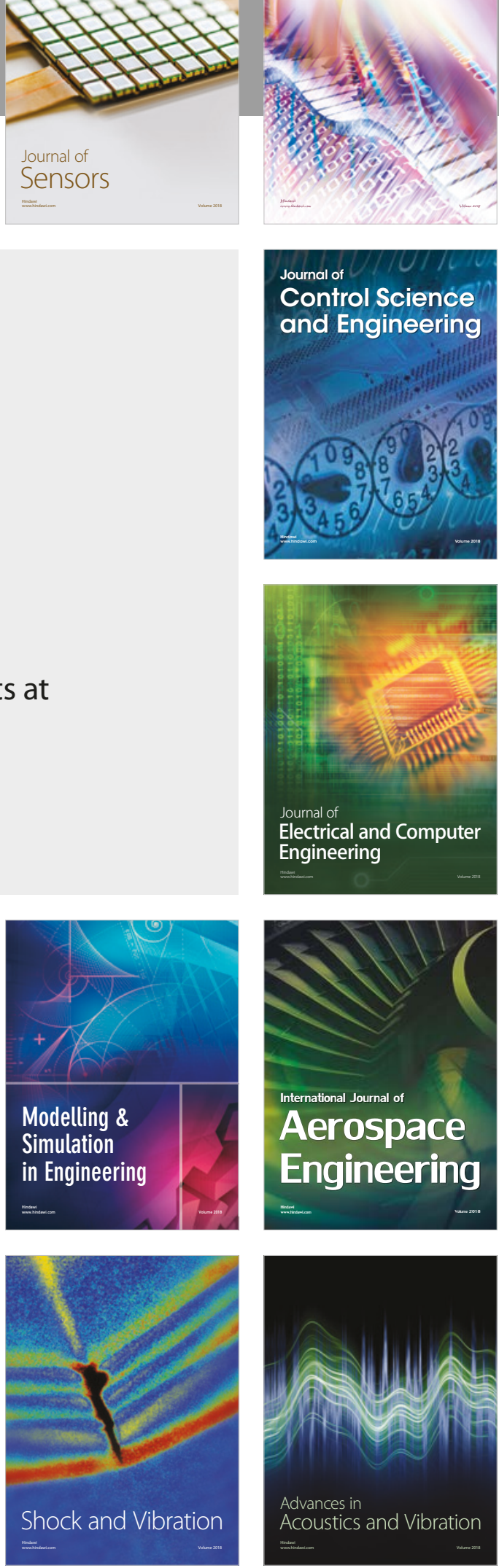